\title{
Interfacial Bonding Mechanism and Mechanical Performance of Continuous Fiber Reinforced Composites in Additive Manufacturing
}

\author{
Congze Fan ${ }^{1,2}$, Zhongde Shan ${ }^{1,3^{*}}$, Guisheng Zou², Li Zhan ${ }^{1,4}$ and Dongdong Yan ${ }^{1}$
}

\begin{abstract}
The additive manufacturing of continuous fiber composites has the advantage of a high-precision and efficient forming process, which can realize the lightweight and integrated manufacturing of complex structures. However, many void defects exist between layers in the printing process of additive manufacturing; consequently, the bonding performance between layers is poor. The bonding neck is considered a key parameter for representing the quality of interfacial bonding. In this study, the formation mechanism of the bonding neck was comprehensively analyzed. First, the influence of the nozzle and basement temperatures on the printing performance and bonding neck size was measured. Second, CT scanning was used to realize the quantitative characterization of bonding neck parameters, and the reason behind the deviation of actual measurements from theoretical calculations was analyzed. When the nozzle temperature increased from 180 to $220^{\circ} \mathrm{C}, \mathrm{CT}$ measurement showed that the bonding neck diameter increased from 0.29 to $0.34 \mathrm{~mm}$, and the cross-sectional porosity reduced from $5.48 \%$ to $3.22 \%$. Finally, the fracture mechanism was studied, and the influence of the interfacial bonding quality on the destruction process of the materials was determined. In conclusion, this study can assist in optimizing the process parameters, which improves the precision of the printing parts and performance between the layers.
\end{abstract}

Keywords: 3D printing, Thermoplastic resin, Continuous fiber, Additive manufacturing

\section{Introduction}

Thermoplastic resin is widely used in fused deposition modeling owing to its advantages, such as excellent toughness, optimal dielectric constant, and large damage tolerance [1]. In 1988, Scott Crump proposed fused deposition modeling technology. In the same year, Stratasys launched a commercial printing equipment based on this technology $[2,3]$. The thermoplastic resin was made into filaments, then melted and deposited by a printing nozzle [4]. This material is used to make the non-bearing structure in aerospace, power electronics, automobile

\footnotetext{
*Correspondence: shanzd@cam.com.cn

${ }^{1}$ State Key Laboratory of Advanced Forming Technology and Equipment, Beijing 100044, China

Full list of author information is available at the end of the article
}

manufacturing, and other fields $[5,6]$. Initially, researchers improved mechanical properties by optimizing the process parameters in additive manufacturing $[7,8]$. Subsequently, short fibers were added to the resin matrix via premixing to form high-performance thermoplastic resin composites. The main research includes fiber content and length control, process parameter optimization, and printing accuracy adjustment [9-11]. The tensile strength of the printed component is usually in the range of $10-100 \mathrm{MPa}$, and the modulus of elasticity is usually in the range of $1-9 \mathrm{GPa}[12,13]$. Recently, researchers added continuous fibers with high strength and modulus to resin matrix to manufacture parts whose strength can be comparable to metals $[14,15]$. 
Mori et al. [16] put the printed continuous fiber composite into a hot oven and heated it for $15 \mathrm{~min}$ to improve its mechanical performance. Fischer et al. [17] designed an additional side channel for conveying continuous fibers in a nozzle to realize impregnation. In 2014, Markforged Corporation developed a series of commercial printers, and the thickness of the printing layer was as low as $0.1 \mathrm{~mm}$. The impacts of fiber volume fraction, interlayer strategy, filling pattern, and fiber orientation on the mechanical properties were explored. Furthermore, fiber breakage, fiber pull-out, and delamination failure modes were analyzed during the bearing process [18].

The continuous fiber composite is limited by its interlayer porosity and poor interfacial bonding in additive manufacturing, which inhibits the efficient transmission of load between the matrix and reinforcing fibers, thus reducing the mechanical properties of the printed parts $[19,20]$. To quantitatively evaluate the interfacial bonding quality of the printed parts, in an assumption by Crockett [20], axisymmetric flow was considered the diffusion process of thermoplastic resin. Provided the cross-sectional area is constant, a liquid-solid conversion model can be established to predict the shape of the specimen and gap size between the printing raster, which indicates the quality of the interface. In addition, to predict the growth of the cylindrical bonding neck, Gurrala et al. [21] improved the Frenkel-Eshelby model, and the size of the bonding neck was calculated by printing parameters and filament properties to evaluate the interfacial bonding of the printed parts.

Presently, the interfacial bonding research in additive manufacturing is mainly aimed at pure resin while research on continuous fiber composites remain insufficient $[22,23]$. However, the addition of continuous fibers in printing alters the physical parameters during heat transfer and shrinkage, which affects the resin-diffusion process on the basement and bonding neck size between the printing raster. In this research, the forming mechanism of the interfacial bonding neck during additive manufacturing of continuous fiber composite (AMCFC) was studied. The effective diffusion time was determined by monitoring the temperature during the printing process; subsequently, the dimensionless bonding parameters were calculated theoretically. Moreover, computerized tomography $(\mathrm{CT})$ scanning was employed to detect the internal cross section of the printed specimen, and the actual size of the bonding neck was determined. Furthermore, the difference between the theoretical calculation and actual measurement was analyzed. The influence of nozzle and basement temperatures on the mechanical properties and size of the bonding neck was investigated via mechanical testing. The fracture mechanism of the printed continuous fiber composite was analyzed, and

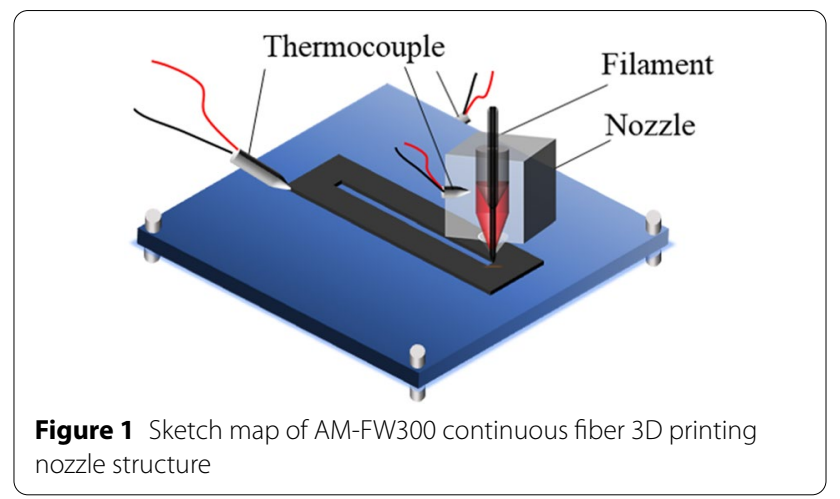

Table 1 3D printing process parameters for specimens

\begin{tabular}{ll}
\hline Parameter & Value \\
\hline Hatch spacing $(\mathrm{mm})$ & 1 \\
Layer thickness $(\mathrm{mm})$ & 0.5 \\
Printing speed $(\mathrm{mm} / \mathrm{s})$ & 5 \\
Diameter of nozzle $(\mathrm{mm})$ & 1 \\
\hline
\end{tabular}

the influence of the interfacial bonding quality on the material fracturing process was also determined. In addition, it was inferred that the interlayer performance of continuous fiber composites can be improved in additive manufacturing by optimizing the process parameters.

\section{Material and Methods}

\subsection{Experimental Materials and Printing Equipment}

In this study, a polylactic acid (PLA) with optimal biodegradability was selected as the resin matrix. It was introduced into the filament printing equipment to impregnate the continuous fibers, then the composite filament with a preset fiber fraction was obtained via melt coating, cooling, winding, and other steps [24]. The AM-FW300 (printing size $300 \times 300 \times 300 \mathrm{~mm}$ ) developed by CAM was used to fabricate the testing specimen. Figure 1 illustrates the printing sketch map while Table 1 presents the printing parameters set to fabricate the specimen.

\subsection{Temperature Monitoring and Property Testing}

K-thermocouples with diameters of $0.02 \mathrm{~mm}$ each were arranged on the printing nozzle, printed basement, surface of the printed parts, and inside the printing chamber to monitor the temperature during printing, as shown in Figure 1. Among them, the K-thermocouples in the printing nozzle moved with the nozzle at the same speed. The thermocouple on the surface of the printed parts was placed on the printed basement and covered with molten material. It monitors the temperature of the first layer. A 
high-frequency analog-to-digital converter Modbus (10 readings per second) was used to collect temperature signals while the upper computer recorded and provided analysis for the measurement results.

The digital image correlation method analyzes the degree of deformation of the samples by comparing the images before and after the deformation. In this study, the VIC-3D XR of Correlated Solutions Company was used to perform the measurement. The US-INSTRON-5567 universal testing machine was used for tensile and flexural testing. Tensile and bending tests were conducted according to the GB/T 1447-2005 and GB/T 1449-2005 standards. The interlaminar shear strength (ILSS) was measured by a three-point bending test according to JCT773-2010. At least five samples, with dimensions of $100 \times 20 \times 3 \mathrm{~mm}$, were tested in each group with a preloading of $5 \mathrm{~N}$ and a loading rate of $5 \mathrm{~mm} / \mathrm{min}$. To obtain the size of the bonding neck, NanoVoxel-3000 CT was adopted to scan the internal structure of the printed specimen. The microstructure of the cross section was analyzed using a JEOL JSM-7500F (10 kV) scanning electron microscope to observe the void and fiber/resin bonding effect.

\section{Results and Discussion}

In the AMCFC process, temperature is a key parameter that influences the bonding process between the printing raster. Therefore, the influence of the nozzle and basement temperatures on the interfacial bonding process was investigated. To further study interfacial bonding, the bonding neck of the printing parts was determined by $\mathrm{CT}$ and temperature monitoring afterwards. Finally, the fracture mechanism with different interfacial bonding necks in AMCFC is discussed.

\subsection{Temperature Monitoring}

In Figure 1, the endpoint of the printed part's short side was selected as the temperature monitoring point, then the effective diffusion time of the material, which refers to the time when the temperature of the material maintains a higher temperature than the critical bonding temperature, was determined via the monitored temperature curve. Figure 2 shows that the temperature at the monitoring point increases swiftly as the printing nozzle approaches, and then decreases rapidly as the nozzle moves away. According to the work by Bellehumeur et al. [25], as the temperature of the deposited material increases beyond the critical bonding temperature, the bonding neck between the printing raster starts to increase. The temperature curve indicates that the material satisfies the conditions of interfacial bonding within the first few seconds of deposition on the basement. When the temperature between the glass transition and critical bonding temperatures is reduced, the material exhibits viscous flow properties and high elasticity, and the interface stops bonding.

Figure 3 presents the temperature variation results at the monitored points under various nozzle and basement temperature conditions. The temperature in the curve increases periodically to the peak, and then abruptly returns to the basement temperature. Figure 3(b) indicates that the first temperature peak at the monitored points occurs at $181{ }^{\circ} \mathrm{C}$, which is slightly lower than the nozzle temperature, then the temperature drops sharply. Although the peak temperature position on the curve is consistent with numerical calculations, the value of the peak temperature is less than that of numerical calculations [26]. The difference in peak temperature originates from the lower monitoring
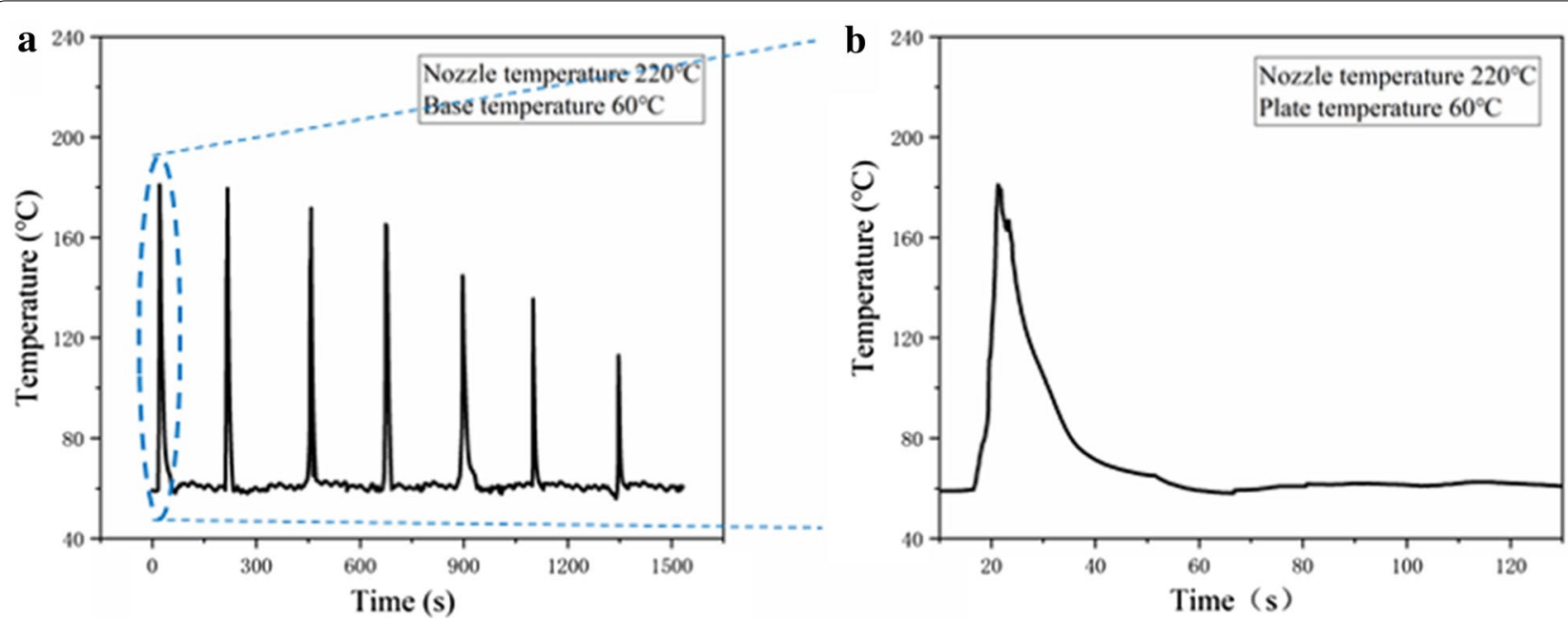

Figure $\mathbf{2}$ a Results of temperature monitored in printing process, $\mathbf{b}$ enlarged view of marked portion in $\mathbf{a}$ 

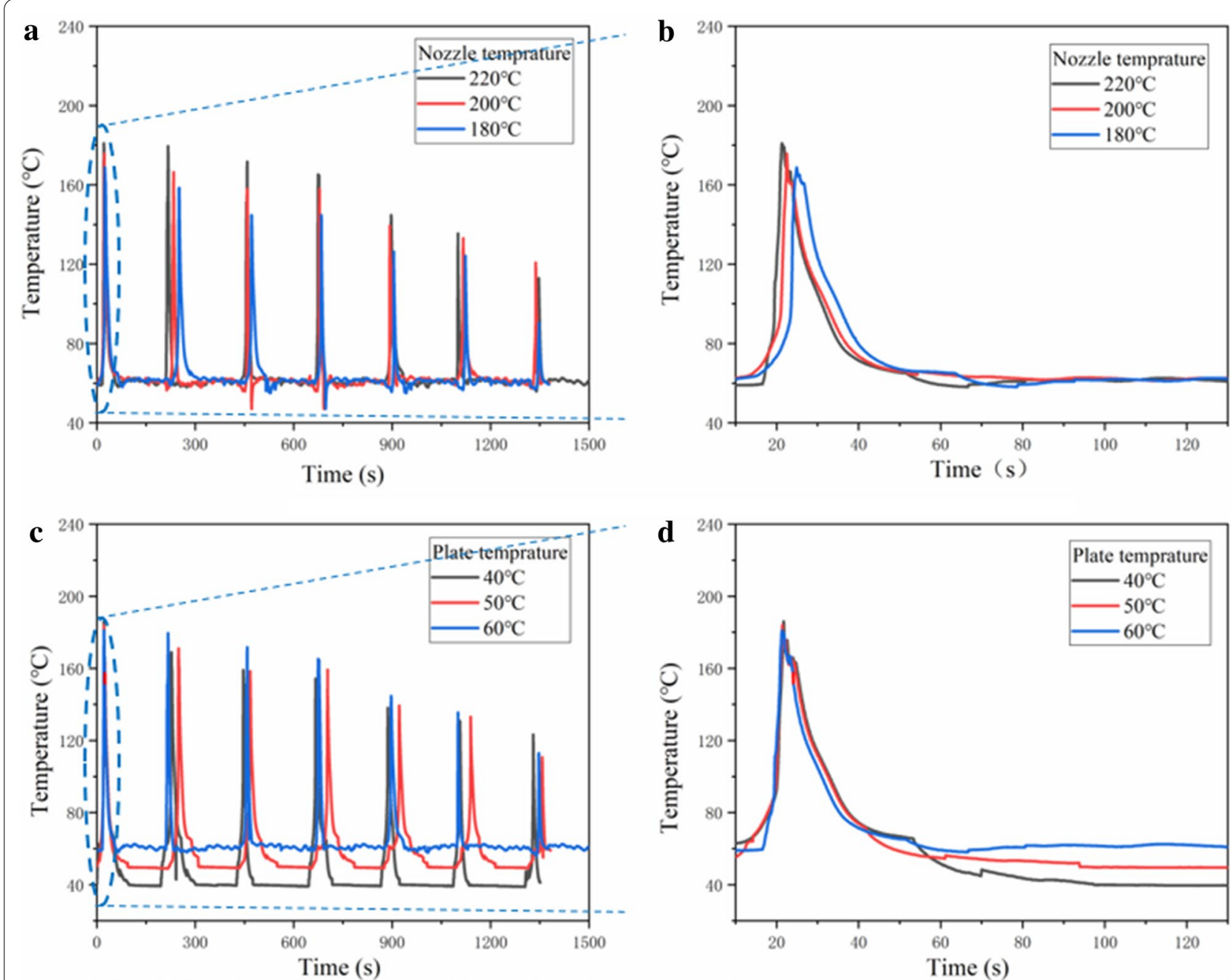

Figure 3 Results of temperature monitored under different nozzle and basement temperatures, where the basement temperature in $\mathbf{a}$ and $\mathbf{b}$ is $60^{\circ} \mathrm{C}$, nozzle temperature in $\mathbf{c}$ and $\mathbf{d}$ is $220^{\circ} \mathrm{C}$, and $\mathbf{b}$ and $\mathbf{d}$ are the enlarged views

frequency, which triggers the true-peak temperature loss during the printing process. As shown in Figure 3(c), as the temperature of the nozzle increases from $180^{\circ} \mathrm{C}$ to $220^{\circ} \mathrm{C}$, the peak value is increased from $169{ }^{\circ} \mathrm{C}$ to $181{ }^{\circ} \mathrm{C}$, and the effective bonding time is increased from $13 \mathrm{~s}$ to $14 \mathrm{~s}$, accordingly. Figure 3(c) and (d) also show that an increase in basement temperature can effectively increase the minimum temperature of the deposition path. At a nozzle temperature of $230{ }^{\circ} \mathrm{C}$ (actual PLA temperature was greater than $230^{\circ} \mathrm{C}$ ), the melt color is altered and thermal degradation occurs; therefore, the highest nozzle temperature is set to $220{ }^{\circ} \mathrm{C}$. In addition, because the cross-sectional shape of the printing raster is irregular, the resin was easily accumulated to a droplet at high temperatures, thus influencing the printing accuracy.

\subsection{Effect of Nozzle Temperature on the Performance of Additive Manufactured Composites}

Figure 4(a) shows the tensile strength of the continuous fiber-reinforced composite specimen. The temperatures of the nozzle were at $180,190,200,210$, and $220{ }^{\circ} \mathrm{C}$, respectively. Although the tensile strength increased monotonically with increasing printing temperature, the increasing percentage initially increased before decreasing. When the nozzle temperature is $220^{\circ} \mathrm{C}$, the average tensile strength is $204.04 \mathrm{MPa}$, which is approximately $25 \%$ higher than that of the specimen at $180{ }^{\circ} \mathrm{C}$. Figure 4(b) and (c) present the tensile fracture DIC test results of the specimens at printing temperatures of 180 and $220{ }^{\circ} \mathrm{C}$, respectively. The position of the red dotted line in Figure 4(b) indicates the fracture interface of the specimen. At a printing temperature of $180^{\circ} \mathrm{C}$, the crack is initially propagated perpendicular to the direction 


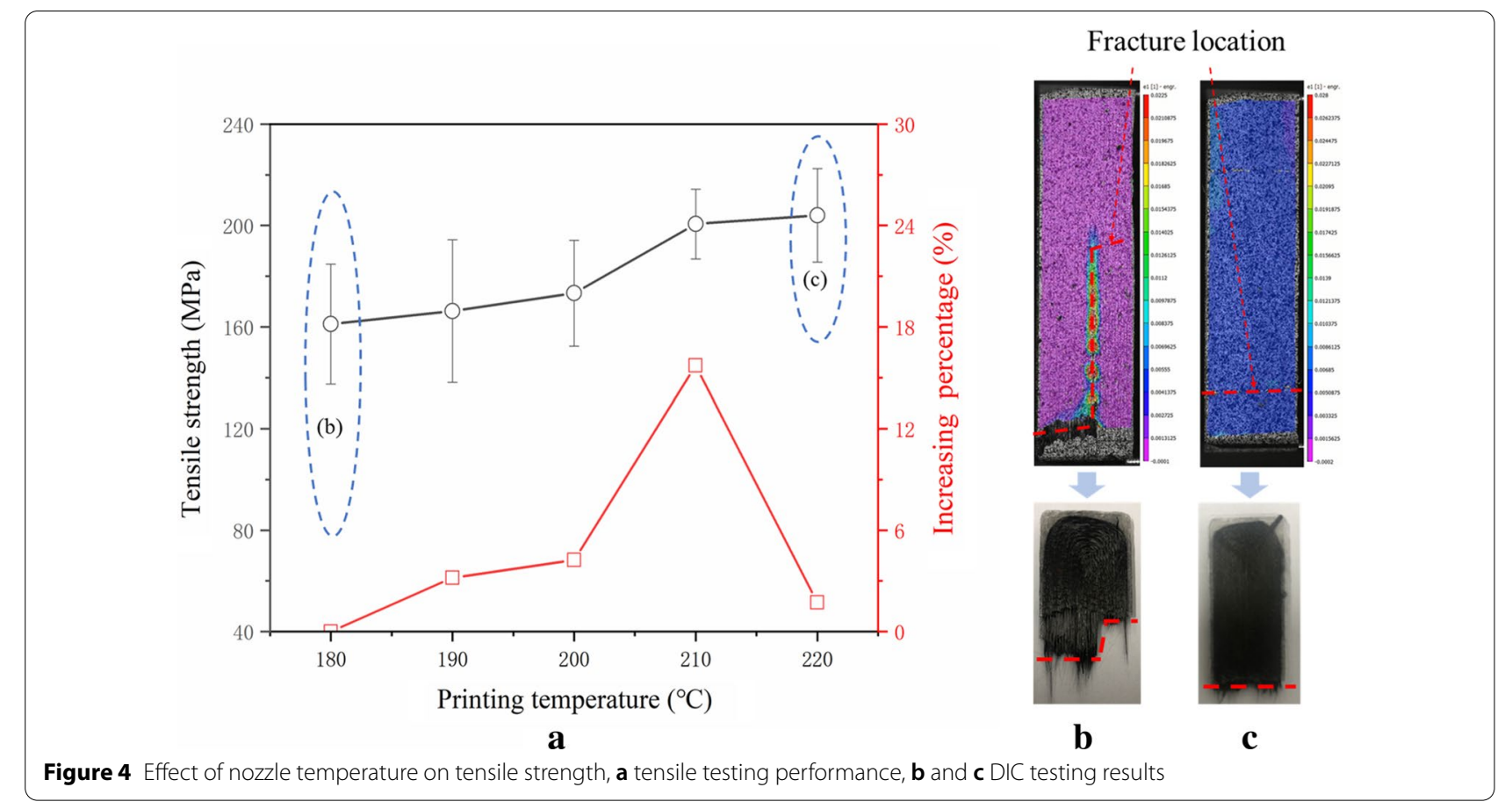

of the fiber, then in parallel to the direction of the fiber, which leads to cracks in the printing raster and interlaminar delamination of the specimen. At the point where the nozzle temperature increases to $220{ }^{\circ} \mathrm{C}$, the fracture interface in Figure 5(c) is perpendicular to the fiber direction, the direction is not altered during crack propagation, and no delamination exists between the layers and printing raster.

In Figure 5(a), when the printing nozzle temperature is $220{ }^{\circ} \mathrm{C}$, the interlayer shear strength is $24.73 \mathrm{MPa}$, which is approximately $70 \%$ higher than that at $180{ }^{\circ} \mathrm{C}$.
The percentage increase of the ILSS attained an extreme value of $28 \%$ at $190{ }^{\circ} \mathrm{C}$, and then gradually decreased. Figure 5(b) shows the stress-strain curve of the ILSS test at printing temperatures of 180 and $220^{\circ} \mathrm{C}$, respectively. At a printing temperature of $220^{\circ} \mathrm{C}$, the tensile stress of the specimen increases to its maximum value faster than that at $180{ }^{\circ} \mathrm{C}$. In addition, although no immediate brittle fracture exists, the specimens yield after reaching the maximum tensile strength.

Thermoplastic resin has a high melting temperature, high viscosity, and poor wettability to continuous fibers,
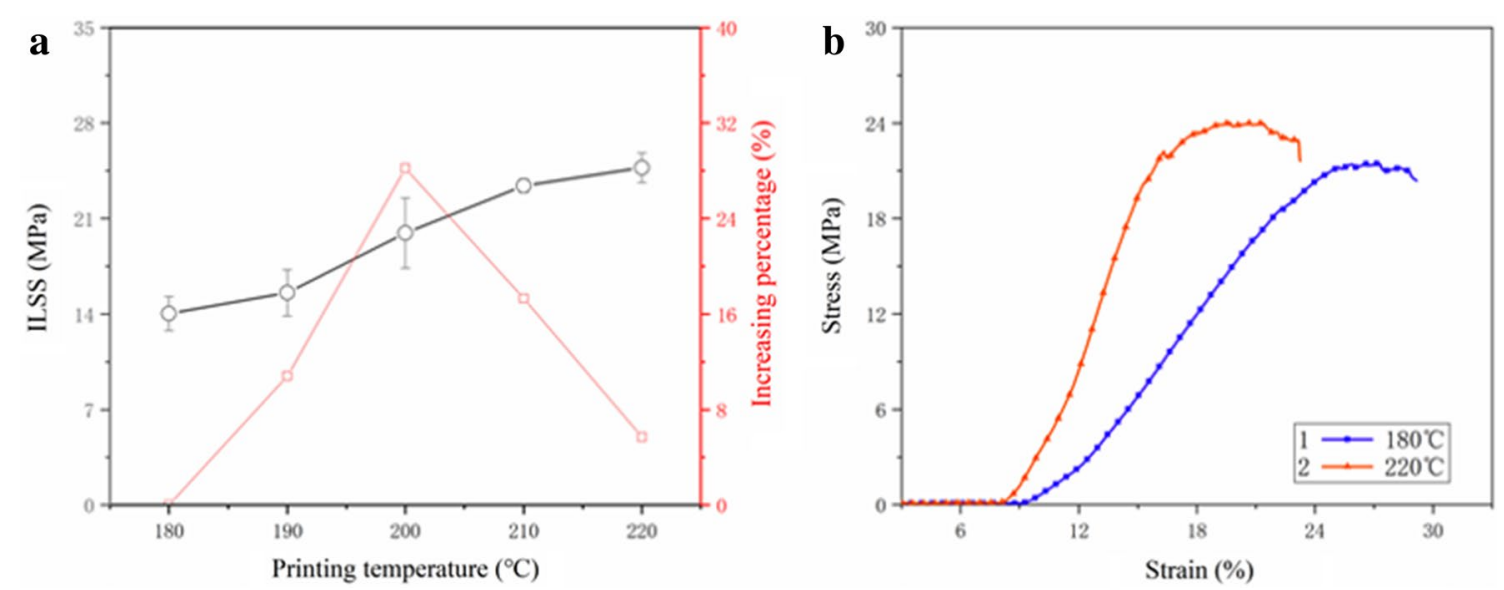

Figure 5 Effect of nozzle temperature on ILSS: a ILSS value variation, $\mathbf{b}$ stress-strain curve 

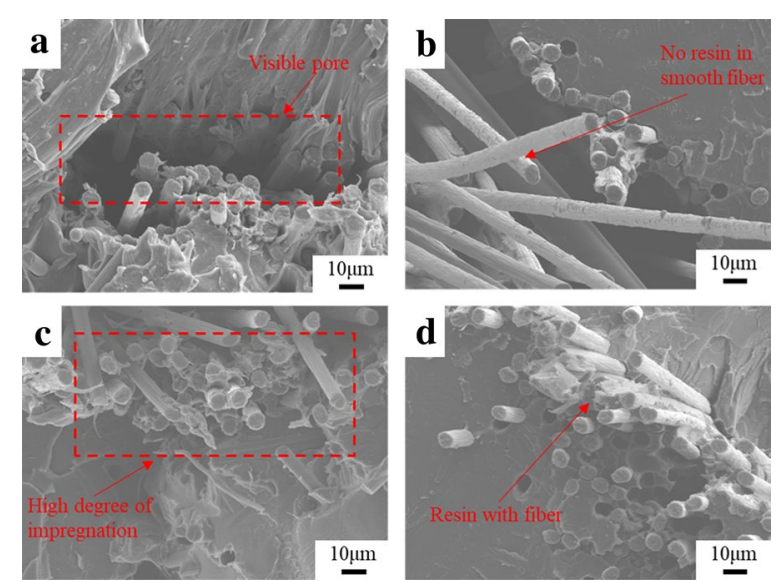

Figure 6 Fracturing-interface microstructures of the cross section at various nozzle temperatures after tensile tests: $\mathbf{a}$ and $\mathbf{b} 180^{\circ} \mathrm{C}, \mathbf{c}$ and d $220^{\circ} \mathrm{C}$

which leads to a low degree of fiber impregnation, as well as poor interfacial bonding properties. Figure 6 illustrates the fracture-interface microstructures of the cross section at different nozzle temperatures. At a nozzle temperature of $180{ }^{\circ} \mathrm{C}$, visible pores appear after extracting the fibers, as shown in Figure 6(a). In addition, a small amount of fibers at the edge are impregnated with resin and remain in the matrix after cracking. In Figure 6(b), a large number of continuous fibers with smooth surfaces can be observed, and no coagulated resin remains on the fiber. When the nozzle temperature increased to $220{ }^{\circ} \mathrm{C}$, the fluidity of the molten resin improved. In Figure 6c) and (d), the continuous fiber exhibits a superior impregnation degree. Here, the continuous fiber is covered with resin, and the interface exhibits perfect bonding. There is no significant delamination in the interfacial cross. Moreover, although numerous continuous fiber breakages occurred during the fracturing of the specimen, it barely separated from the matrix.

\subsection{Effect of Basement Temperature on the Performance of Additive Manufactured Composites}

Figure 3(c) and (d) show that the basement temperature determines the minimum temperature of the specimen and also influences temperature distribution during the printing process. ISLL and flexural strength are the most sensitive properties to the change in adhesion degree at the interface. Figure 7 illustrates the three-point bending test specimen with basement temperatures of 40,50, and $60{ }^{\circ} \mathrm{C}$, respectively. In Figure 8, when the basement temperature increases from $40{ }^{\circ} \mathrm{C}$ to $60^{\circ} \mathrm{C}$, the average interlayer shear strength increases from 23.59 to $24.72 \mathrm{MPa}$, increasing by $5 \%$. At a $5 \%$ increase from 344.30 to 350.21 $\mathrm{MPa}$, the increase in the average flexural strength is

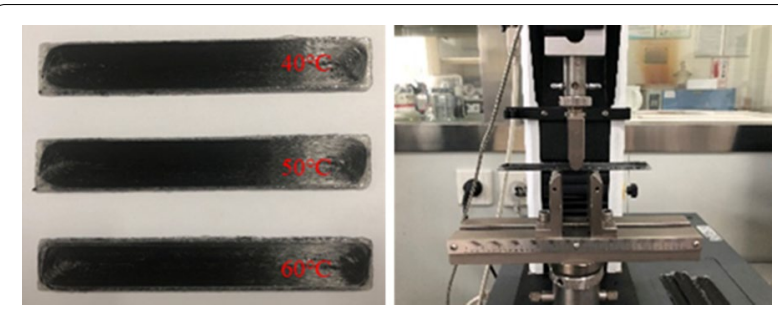

Figure 7 Testing specimens and three-point bending test process

insignificant. In summary, the experiments verify that the basement temperature exhibits an insignificant effect on the interfacial structure and performance of the printed parts.

\subsection{Formation and Calculation of Interfacial Bonding Neck} During the fused deposition process, the continuous fibers were coated with resin, and the printing raster remained bonded by the resin. Heat was the driving force that fused and bonded the materials [27]. The bonding process between the printing raster includes surface approaching, surface contact (wetting and diffusion processes), and neck formation. Wetting starts as soon as the surfaces are in contact, and the molecular diffusion barrier between the interfaces of the molten resin disappears. By the end of the surface contact stage, the molecular chains move freely through the interface to form a bonding neck, and then the interfacial bonding is terminated $[28,29]$.

\subsubsection{Formation Mechanism of Bonding Neck}

Figure 9 illustrates the formation process of the bonding neck during the printing process. The bonding neck is the interface located where two printing roads meet, and it is the most critical surface where bond strength is developed. The size of the bonding neck is always smaller than that of the printing width. For the incompressible fluid, the Frenkel-Eshelby model was established by modifying the Frenkel model [30]. When the processing temperature reaches the critical bonding temperature, two identical spherical particles coalesce in the viscous flow. The dimensionless bonding parameter $C$ is the ratio of the neck radius y to the composite radius $a$, as expressed below:

$$
C=\frac{y}{a}=\left(\frac{\Gamma t}{\mu a_{0}}\right)^{\frac{1}{2}},
$$

where $a, a_{0}, y, t, \mu$ and $\Gamma$ represent the particle radius, bonding neck diameter, bonding time, viscosity, and surface tension, respectively. In addition, the printing rate, printing length, printing time, and raster width also affect 

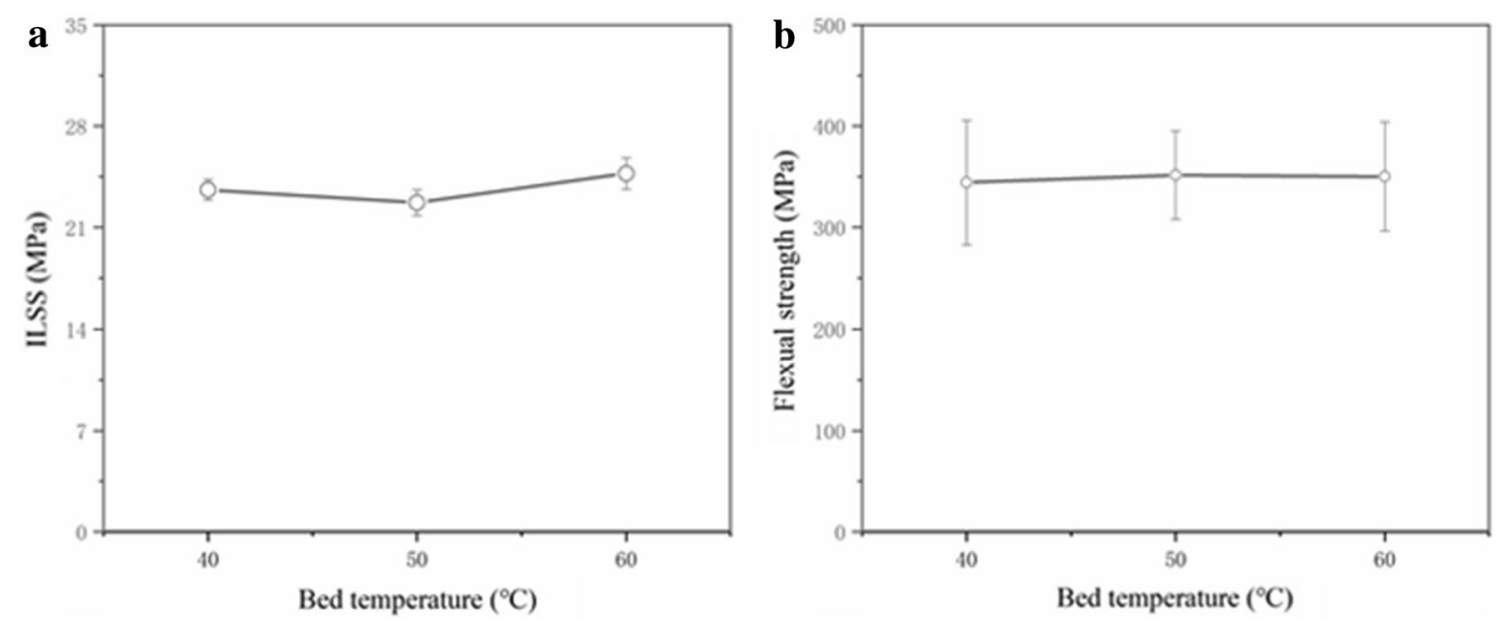

Figure 8 ILSS and flexural strength at different basement temperatures

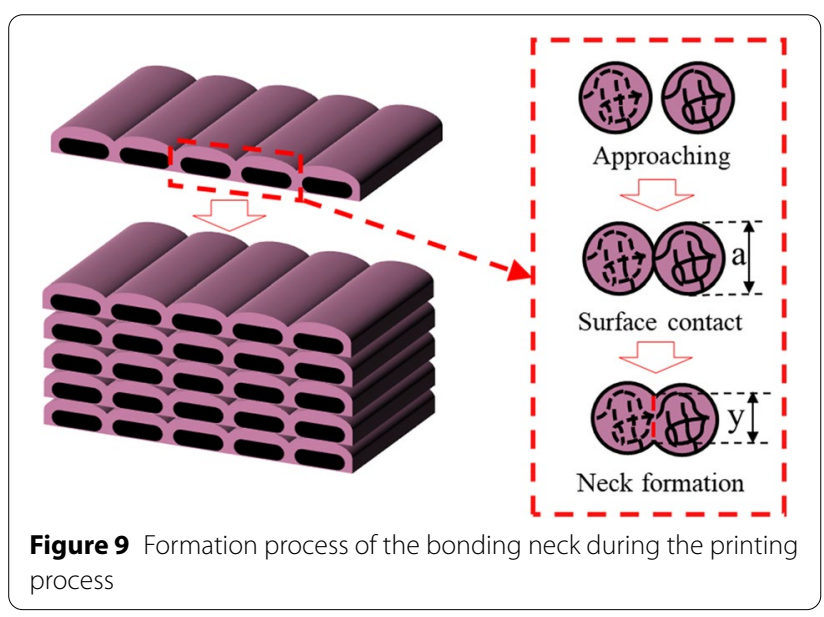

the coalescence between resin paths. Considering the total printing time and layer thickness, a modified Frenkel-Eshelby model was obtained [31], and the dimensionless bonding parameter $C$ can be expressed as:

$$
C=\frac{y}{a}=\left(\frac{\Gamma \frac{t_{a}}{l m n}}{\mu \frac{\mathrm{h}}{n}}\right)^{\frac{1}{2}}=\left(\frac{\Gamma t_{a}}{\mu j h n}\right)^{\frac{1}{2}},
$$

where $t_{a}, m, l, j, h$ and $n$ are the effective bonding time, number of printing raster, printing length, ratio of the number of printing raster to number of layers, printing height, and number of layers, respectively. Viscosity and surface tension are assumed to be constant in this study. The size of the bonding neck between the printing raster can be calculated using the Frenkel-Eshelby model.

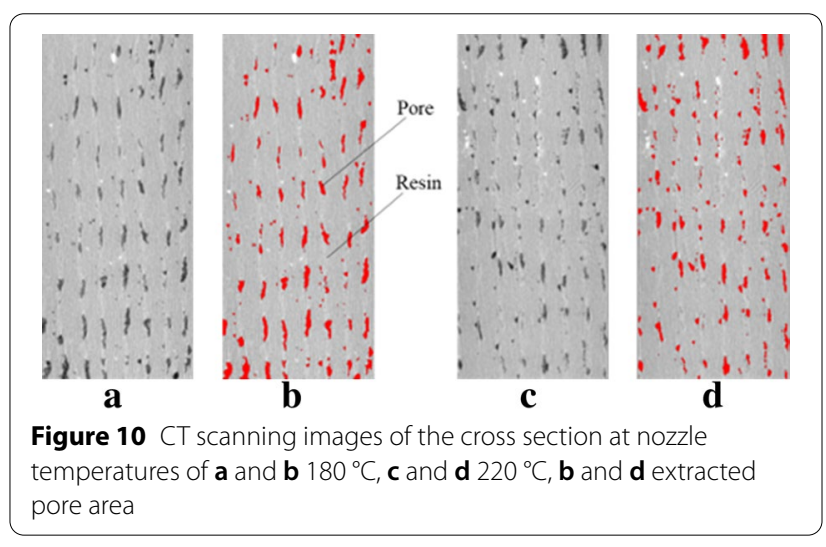

\subsubsection{Calculation of Interfacial Bonding Neck}

In tensile or flexural tests, when the specimen is fractured, the failure interface generates plastic deformation, altering the structural size of the interface. Consequently, it cannot be adopted in calculating the size of the bonding neck. In addition, a large number of continuous fibers exist in the cross section after fracturing, which will affect the measurement and characterization of pores. Therefore, to determine the size of the cross section in this study, CT technology was adopted to scan the printed specimen. Figure 10 presents the CT scanning images of the specimen at printing nozzle temperatures of $180{ }^{\circ} \mathrm{C}$ and $220{ }^{\circ} \mathrm{C}$. It can be observed that the bonding neck inside the printing cross section is inconsistent. To avoid the edge effect, the diameter of the bonding neck was measured from the middle layer of the sample. Five groups of bonding neck dimensions were measured, and their average value was used to calculate the dimensionless bonding parameters $(y / a)$. The red parts in Figures 10(b) and (d) represent the pores, 
which are also used in calculating the porosity of the cross section and to indicate the quality of the interfacial bonding.

The performance parameters of the thermoplastic resin are presented in Table 2. These parameters are used to calculate the theoretical bonding neck via the mathematical model. Table 3 presents the theoretical and experimental values of the bonding neck. When the printing temperature is $180{ }^{\circ} \mathrm{C}$, the experimentally measured bonding neck diameter is $0.29 \mathrm{~mm}$, and the dimensionless bonding parameter is 0.58 , which is greater than the 0.14 value calculated by the theoretical model. At a printing temperature of $220^{\circ} \mathrm{C}$, the experimentally measured bonding neck diameter is increased to $0.34 \mathrm{~mm}$, and the dimensionless bonding parameter to 0.67 , with an increase of approximately $15 \%$. In addition, as the nozzle temperature increases, the cross section in Figure 10 exhibits an increase in the degree of diffusion, as the interlayer pores shrink, and the bonding neck size increases. At a nozzle temperature of $220^{\circ} \mathrm{C}$, the porosity of the cross section decreases to $3.22 \%$ by approximately $40 \%$, which is the same as that of the bonding neck size. Furthermore, when the printing temperature is 180 or $220{ }^{\circ} \mathrm{C}$, the value of the dimensionless bonding parameters predicted by the theoretical model is less than the experimental value, which indicates that the theoretical model underestimates the diameter of the bonding neck between the printing raster.

\subsection{Discussion on Interfacial Fracture Mechanism}

The effect of temperature on the performance of the composites was studied, and the formation mechanism of the interfacial bonding neck was determined. Therefore, it is necessary to establish the relationship between temperature and interfacial bonding neck size. The fracture mechanism with different interfacial bonding necks

Table 2 Performance parameters of the thermoplastic resin

\begin{tabular}{ll}
\hline Parameter & Value \\
\hline Viscosity (Pa.s) & 1500 \\
Surface tension (N/m) & 0.029 \\
\hline
\end{tabular}

needs to be established to assist in optimizing the process parameters and improving the precision of printing parts, as well as the performance between the layers.

\subsubsection{Effect of Temperature on Interfacial Bonding}

In Figure 4, when the temperature of the printing nozzle increases from $180{ }^{\circ} \mathrm{C}$ to $220^{\circ} \mathrm{C}$, the fracture failure between the printing raster and printing layer under tensile load is reduced. By combining the results of the ILSS test presented in Figure 5, the interlayer performance of the material and bonding quality within the layer are improved as the temperature of the nozzle increases. In addition, at a nozzle temperature of $220{ }^{\circ} \mathrm{C}$ (Figure 6), the degree of impregnation between the fibers and the resin is high, the fiber is evenly coated with resin, and the bonding quality between the fibers and resin is superior. The composite material is deposited on the previous printing raster, and heat is transferred to the formed parts via thermal conduction, such that the temperature of the forming position is higher than the critical bonding temperature [32]. Additionally, the bonding neck is formed between the adjacent printing raster. According to the data in Table 3, when temperature increases to $220^{\circ} \mathrm{C}$, the diameter of the bonding neck increases by approximately $15 \%$. Combined with the above mechanical performance test results, it is evident that the bonding neck is closely related to the interlayer shear performance. The size of the bonding neck can be used to evaluate the bonding quality of printing. As the nozzle temperature increased, the diameter of the bonding neck increased, the gap between the layers and the printing raster decreased, the interfacial bonding quality improved, and ILSS also improved. In summary, the bonding strength of the specimen can be improved by increasing the temperature of the extrusion nozzle, and excellent mechanical properties can also be obtained.

In Table 3, the theoretical model significantly underestimates the size of the bonding neck between adjacent printing rasters. When Eq. (2) is used to predict the bonding degree between two printing paths, the interferential factors are not considered. On one hand, when the molten PLA is deposited away from the nozzle, an elliptical cross section is obtained; however, it is assumed to be circular in the mathematical model without considering the change in shape. On the other hand, the presence of

Table 3 Theoretical and experimental values of the bonding neck of printed parts

\begin{tabular}{|c|c|c|c|c|c|c|}
\hline \multirow{2}{*}{$\begin{array}{l}\text { Nozzle temperature } \\
\left({ }^{\circ} \mathrm{C}\right)\end{array}$} & \multirow{2}{*}{$\begin{array}{l}\text { Bonding neck diameter } \\
(\mathrm{mm})\end{array}$} & \multicolumn{3}{|c|}{ Dimensionless bonding parameter $(y / a)$} & \multicolumn{2}{|c|}{ Porosity (\%) } \\
\hline & & Experimental & St. dev. & Theoretical & Average & St. dev. \\
\hline 180 & 0.29 & 0.58 & 0.05 & 0.14 & 5.48 & 0.17 \\
\hline 220 & 0.34 & 0.67 & 0.06 & 0.15 & 3.22 & 0.31 \\
\hline
\end{tabular}


continuous fibers will alter the thermal stress field, which will affect the formation of the bonding neck. Fibers with low deformation will cause the thermoplastic resin to move to both sides of the printing raster, thereby increasing the size of the bonding neck. The difference between the model prediction and experimental test may also originate from the creep deformation of the resin under gravity. In addition, the diameter of the bonding neck between adjacent printing raster is not the same at different positions in the $\mathrm{CT}$. The size of the bonding neck at the top layer is smaller and the bonding quality is poorer. This is owing to the difference in the cooling rate between the top and bottom layers, as the bottom layer undergoes a longer high-temperature process (greater than the critical bonding temperature) than the top layer.

\subsubsection{Fracture Mechanism}

In AMCFC, the resin and continuous fiber represent the matrix and reinforcing phase, respectively. The bonding neck formed by molecular diffusion and polymer chain crosslinking is a key parameter for evaluating the quality of the interface. Interfacial bonding consists of two phases: intra-layer and inter-layer bonding. As shown in Figure 11, intra-layer bonding is the bonding between adjacent printing raster, whereas inter-layer bonding is defined as the bonding between two consecutive layers. The quality of both intra-layer and inter-layer bonding affects the fracture mode of the composite material.

The fracture mode is the external manifestation of the microstructure at the interface. The quality of the interfacial bonding in the micro-regions between the resin raster, as well as between the resin and fiber, directly determines the fracture mechanism of the printed parts under tensile load. Figure 12 illustrates the main fracture mode of the continuous fiber-reinforced composite. When the diameter of the bonding neck is small, the interfacial bonding quality is poor, and the main failure

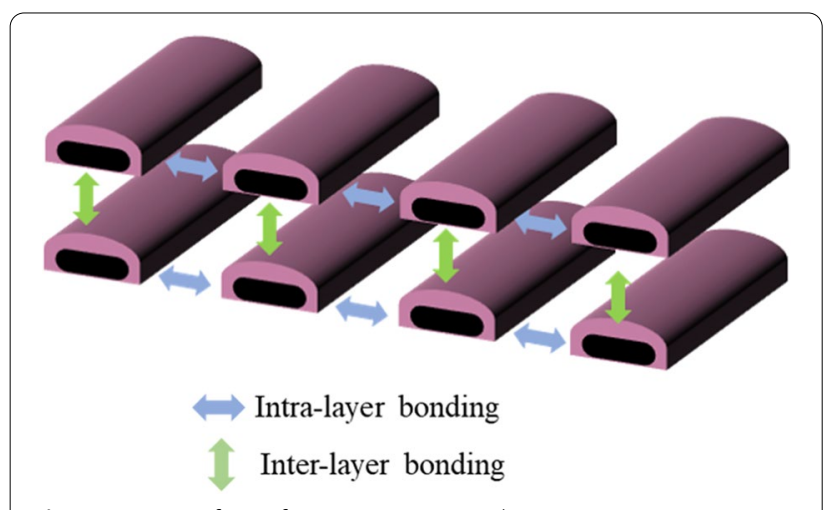

Figure 11 Interface of composite materials in printing process

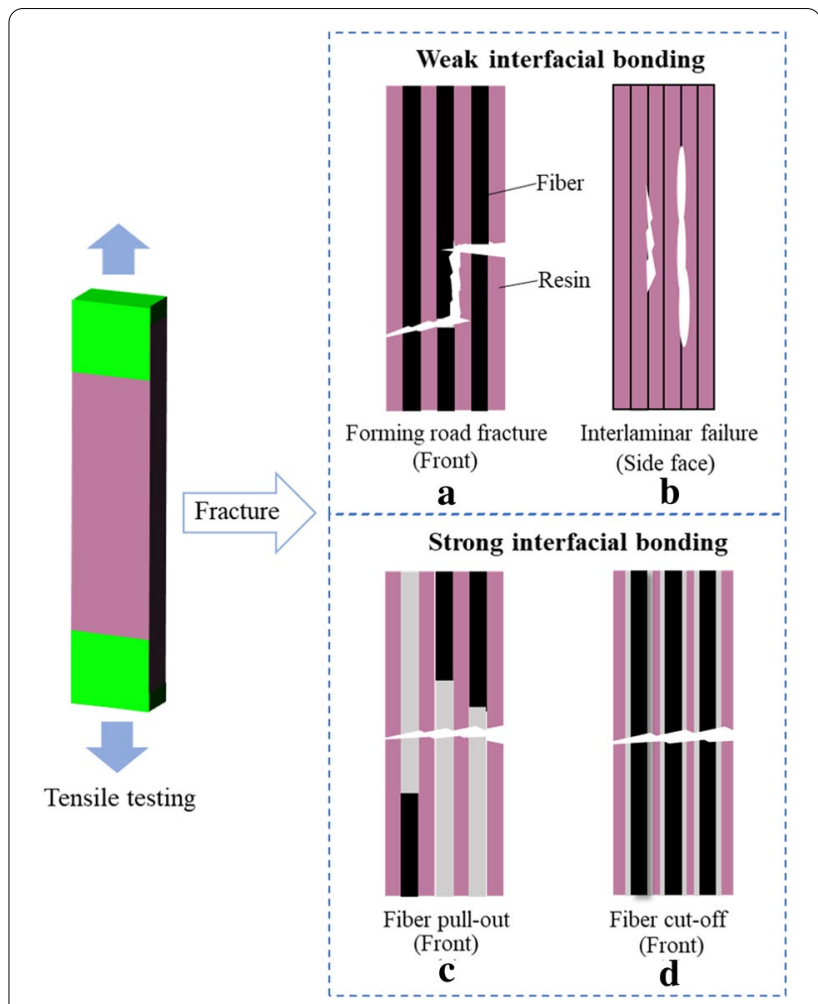

Figure 12 Main fracture mode of AMCFC during tensile test

mode is the unbalanced separation between the printing raster and delamination between the printed layers, as shown in Figure 12(a) and (b). The bonding neck diameter of the specimen, as shown in Figure 4(b), is $0.29 \mathrm{~mm}$, and the bonding quality inside the layers is poor. Consequently, during the stretching process, the crack quickly turns parallel to the printing raster after encountering the weak bonding positions between the printed raster until the material breaks owing to the crack expansion, as shown in Figure 12(a). When large numbers of pores exist between layers or the bonding quality is poor, the tensile stress will cause delamination between layers, as shown in Figure 12(b).

When the diameter of the bonding neck is large and the interfacial bonding quality is excellent, defects and stress concentration no longer occur in the printing raster and printed layer, but fracture occurs at the middle of the printed specimen under tensile stress, as shown in Figure 4(c). In Figure 12(c) and (d), the crack propagation direction in the printed par is hardly altered, it only expands linearly perpendicular to the fiber interface, and exhibits a flat fracture. In Figure 13, the impregnation degree between the fiber and resin is a key parameter that affects the quality of the interfacial bonding and determines the failure process of the composite material. When the degree of impregnation is low, the 


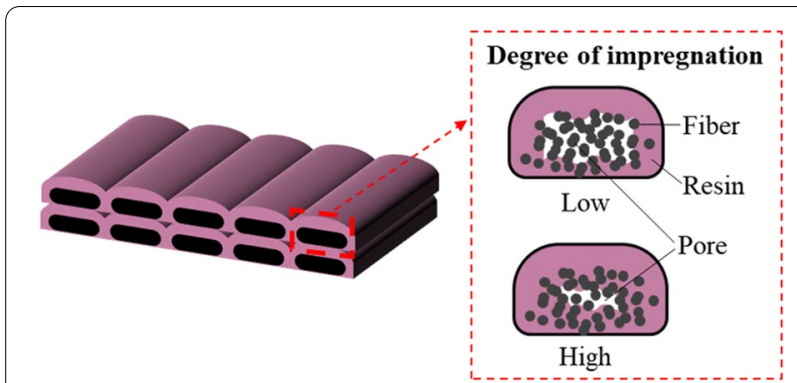

Figure 13 Continuous fiber composite with different impregnation degree

interface quality between the fiber and resin is poor, as shown in Figure 6(a) and (b). Consequently, load cannot be transferred to the fiber bundle when the matrix material cracks. Large numbers of continuous fibers are finally pulled out after fracturing, as shown in Figure 12(c); therefore, the mechanical properties are poor. When the fibers are highly impregnated with the matrix, the bonding strength between the fiber and resin interface is high, as shown in Figure 6(c) and (d). Therefore, stress can be smoothly transferred from the matrix to the fiber through the interface. During crack propagation, continuous fibers exhibit a "barrier effect", as shown in Figure 12(d). The external loads are used to perform the surface work, and the composite is more difficult to damage according to the conservation of energy. Therefore, "barrier effect" indicates an obstacle to the crack, which leads to challenges during propagation. The fibers break with further increase in stress; subsequently, the resin matrix breaks with high strain.

\section{Conclusions}

Aimed at addressing the interfacial bonding challenge in $\mathrm{AMCFC}$, the following conclusions were drawn from this study via experimental research and theoretical calculations:

(1) When the nozzle temperature increased from $180{ }^{\circ} \mathrm{C}$ to $220{ }^{\circ} \mathrm{C}$, the tensile strength increased by $25 \%$ to $204.04 \mathrm{MPa}$, and the ILSS increased by approximately $70 \%$ to $24.73 \mathrm{MPa}$. The improved performance is attributed to the increase in the impregnation degree between the fiber and resin, as well as the enhanced interfacial bonding.

(2) When the nozzle temperature increased from $180{ }^{\circ} \mathrm{C}$ to $220^{\circ} \mathrm{C}$, the $\mathrm{CT}$ measurement indicated that the bonding neck diameter increased from 0.29 to $0.34 \mathrm{~mm}$ while the cross-sectional porosity reduced from $5.48 \%$ to $3.22 \%$. Combined with the mechanical properties, the formation mechanism of the bonding neck was analyzed to evaluate the bonding quality of the printing process.

(3) The fracture mechanism of AMCFC depends on the quality of the interfacial bonding. The degree of impregnation between the fiber and resin significantly affects the fracture mode. Continuous fibers with high impregnation degree can exhibit a "barrier" effect on crack propagation; Consequently, better mechanical performance is obtained.

\section{Acknowledgements}

Not applicable.

\section{Authors' Contributions}

CF was in charge of the entire study and prepared the manuscript; ZS supervised and reviewed this study, and he acquired the funding; GZ guided the experiments; LZ and DY contributed to methodology and software. All authors have read and approved the final manuscript.

\section{Authors' Information}

Congze Fan, born in 1990, is currently a PhD candidate at Department of Mechanical Engineering, Tsinghua University, China. He received his master's degree from Harbin University of Science and Technology, China, in 2015. His research interests include additive manufacturing and composites.

Zhongde Shan, born in 1970, is currently the President of Nanjing University of Aeronautics and Astronautics, China. He received his PhD degree in mechanical engineering from Tsinghua University, China, in 2002.

Guisheng Zou, born in 1966, is currently a professor at Department of Mechanical Engineering, Tsinghua University, China.

Li Zhan, born in 1980, is currently a professor at State Key Laboratory of Advanced Forming Technology and Equipment, China Academy of Machinery Science \& Technology Group Co., Ltd., China.

Dongdong Yan, born in 1994, received his master's degree from State Key Laboratory of Advanced Forming Technology and Equipment, China Academy of Machinery Science \& Technology Group Co., Ltd., China.

Funding

Supported by National Key R\&D Program of China (Grant No. 2017YFB1103400).

\section{Competing Interests}

The authors declare no competing financial interests.

\section{Author Details}

${ }^{1}$ State Key Laboratory of Advanced Forming Technology and Equipment, Beijing 100044, China. ${ }^{2}$ Department of Mechanical Engineering, Tsinghua University, Beijing 100084, China. ${ }^{3}$ Nanjing University of Aeronautics and Astronautics, Nanjing 210016, China. ${ }^{4}$ China Academy of Machinery Science \& Technology Group Co., Ltd., Beijing 100044, China.

Received: 9 May 2020 Revised: 22 November 2020 Accepted: 13 January 2021

Published online: 15 February 2021

\section{References}

[1] A N Dickson, J N Barry, K A Mcdonnell, et al. Fabrication of continuous carbon, glass and Kevlar fiber reinforced polymer composites using additive manufacturing. Additive Manufacturing, 2017, 16: 146-152. 
[2] C Vălean, L Marșavina, M Mărghitaș, et al. Effect of manufacturing parameters on tensile properties of FDM printed specimens. Procedia Structural Integrity, 2020, 26: 313-320.

[3] L Wang, W M Gramvlich, D J Gardner. Improving the impact strength of Poly (lactic acid)(PLA) in fused layer modeling (FLM). Polymer, 2017, 114: 242-248.

[4] B M Lorenzo, A Díaz, I I Cuesta. Influence of raster orientation on the determination of fracture properties of polypropylene thin components produced by additive manufacturing. Theoretical and Applied Fracture Mechanics, 2020, 107: 102536.

[5] I Hugo, J Zaragoza-Siqueiros. Design and manufacturing strategies for fused deposition modelling in additive manufacturing: A review. Chinese Journal of Mechanical Engineering, 2019, 32: 53.

[6] H L Tekinalp, V Kunc, G M Velez-Garcia, et al. Highly oriented carbon fiberpolymer composites via additive manufacturing. Composites Science and Technology, 2014, 105: 144-150.

[7] D Espalin, R J Alberto, F Medina, et al. Multi-material, multi-technology FDM: exploring build process variations. Rapid Prototyping Journal, 2014, 20(3): 236-244.

[8] B Huang, S Singamneni. Raster angle mechanics in fused deposition modelling. Journal of Composite Materials, 2015, 49(3): 363-383.

[9] F Ning, W Cong, J Qiu, et al. Additive manufacturing of carbon fiber reinforced thermoplastic composites using fused deposition modeling. Composites Part B Engineering, 2015, 80: 369-378.

[10] G Liao, Z Li, Y Cheng, et al. Properties of oriented carbon fiber/polyamide 12 composite parts fabricated by fused deposition modeling Materials \& Design, 2018, 139: 283-292.

[11] M Somireddy, CV Singh, A Czekanski. Mechanical behaviour of 3D printed composite parts with short carbon fiber reinforcements. Engineering Failure Analysis, 2020, 107: 104232.

[12] J Wang, H Xie, Z Weng, et al. A novel approach to improve mechanical properties of parts fabricated by fused deposition modeling. Materials \& Design, 2016, 105: 152-159.

[13] S Martin, S Chethan, A Florian, et al. Anisotropic properties of oriented short carbon fibre filled polypropylene parts fabricated by extrusionbased additive manufacturing. Composites Part A: Applied Science and Manufacturing, 2018, 113: 95-104.

[14] XTian, T Liu, C Yang, et al. Interface and performance of 3D printed continuous carbon fiber reinforced PLA composites. Composites Part A: Applied Science and Manufacturing, 2016, 88: 198-205.

[15] N Li, Y Li, S Liu. Rapid prototyping of continuous carbon fiber reinforced polylactic acid composites by 3D printing. Journal of Materials Processing Technology, 2016, 238: 218-225.

[16] K Mori, T Maeno, Y Nakagawa. Dieless forming of carbon fibre reinforced plastic parts using 3D printer. Procedia Engineering, 2014, 81: 1595-1600.

[17] A Fischer, S Rommel, T Bauernhansl. New fiber matrix process with 3D fiber printer-A strategic in-process integration of endless fibers using fused deposition modeling (FDM). IFIP International Conference on Digital Product and Process Development Systems, 2013: 167-175.

[18] M A Caminero, J M Chacón, I García-Moreno, et al. Impact damage resistance of 3D printed continuous fibre reinforced thermoplastic composites using fused deposition modelling. Composites Part B Engineering, 2018, 148: 93-103.

[19] Z Shan, C Fan, Q Sun, et al. Research on additive manufacturing technology and equipment for fiber reinforced resin composites. China Mechanical Engineering, 2020, 31(2): 221-226. (in Chinese)

[20] R S Crockett. The liquid-to-solid transition in stereodeposition techniques Solid Freeform Fabrication Proceedings. Austin: University of Texas, 1997: 257-264.

[21] P K Gurrala, S P Regalla. Part strength evolution with bonding between filaments in fused deposition modelling: This paper studies how coalescence of filaments contributes to the strength of final FDM part. Virtual and Physical Prototyping, 2014, 9(3): 141-149.

[22] Y Yan, R Zhang, G Hong, et al. Research on the bonding of material paths in melted extrusion modeling. Materials and Design, 2000, 21(2): 93-99.

[23] P Parandoush, D Lin. A review on additive manufacturing of polymerfiber composites. Composite Structures, 2017, 182: 36-53.

[24] C Fan, Z Shan, G Zou, et al. Forming laws of continuous fiber composite filaments in 3D printing. China Mechanical Engineering, 2020, 30(9): 10891097. (in Chinese)

[25] C Bellehumeur, L Li, Q Sun, et al. Modeling of bond formation between polymer filaments in the fused deposition modeling process. Journal of Manufacturing Processes, 2004, 6(2): 170-178.

[26] DYWu, S Meure, D Solomon. Self-healing polymeric materials: a review of recent developments. Progress in Polymer Science, 2008, 33(5): 479-522.

[27] L Li, Q Sun, C Bellehumeur, et al. Composite modeling and analysis for fabrication of FDM prototypes with locally controlled properties. Journal of Manufacturing Processes, 2002, 4(2): 129-141.

[28] S F Costa, F M Duarte, J A Covas. Towards modelling of Free Form Extrusion: analytical solution of transient heat transfer. International Journal of Material Forming, 2008, 1(1): 703-706.

[29] E Barocio, B Brenken, A Favaloro, et al. Fusion bonding simulations of semi-crystalline polymer composites in the extrusion deposition additive manufacturing process. American Society for Composites, 2017.

[30] J F Rodriguez, J P Thomas, J E Renaud. Mechanical behavior of acryIonitrile butadiene styrene fused deposition materials modeling, Rapid Prototyping Journal, 2003, 9(1): 219-230.

[31] Q Sun, G M Rizvi, CT Bellehumeur, et al. Effect of processing conditions on the bonding quality of FDM polymer filaments. Rapid Prototyping Journal, 2008, 14(2): 72-80.

[32] B Huang, S Singamneni. Adaptive slicing and speed- and time-dependent consolidation mechanisms in fused deposition modeling. Proceedings of the Institution of Mechanical Engineers, Part B: Journal of Engineering Manufacture, 2014, 228(1): 111-126.

\section{Publisher's Note}

Springer Nature remains neutral with regard to jurisdictional claims in published maps and institutional affiliations.

\section{Submit your manuscript to a SpringerOpen ${ }^{\odot}$ journal and benefit from:}

- Convenient online submission

- Rigorous peer review

- Open access: articles freely available online

- High visibility within the field

- Retaining the copyright to your article

Submit your next manuscript at springeropen.com 